\title{
Molecules on Insulating Films: Scanning-Tunneling Microscopy Imaging of Individual Molecular Orbitals
}

\author{
Jascha Repp and Gerhard Meyer \\ IBM Research, Zurich Research Laboratory, CH-8803 Rüschlikon, Switzerland \\ Sladjana M. Stojković, André Gourdon, and Christian Joachim \\ CEMES-CNRS, 29 rue J. Marvig, P.O. Box 4347, F-31055 Toulouse Cedex, France \\ (Received 23 September 2004; published 19 January 2005)
}

\begin{abstract}
Ultrathin insulating $\mathrm{NaCl}$ films have been employed to decouple individual pentacene molecules electronically from the metallic substrate. This allows the inherent electronic structure of the free molecule to be preserved and studied by means of low-temperature scanning-tunneling microscopy. Thereby direct images of the unperturbed molecular orbitals of the individual pentacene molecules are obtained. Elastic scattering quantum chemistry calculations substantiate the experimental findings.
\end{abstract}

Scanning-tunneling microscopy (STM) is particularly suited to study the structural environment of an adsorbed molecule on the atomic length scale and to probe the electronic properties of this individual, well-characterized structure. As STM relies on the nonzero conductance of its tunneling junction to produce an image, the majority of the STM studies of individual molecules has so far been limited to molecules on metals or semiconductors. In these cases the electronic structure of the molecules is strongly perturbed by the presence of the substrate electrons. To understand the electronic properties of an individual molecule in mesoscale devices [1] and for monomolecular electronics [2], an electronic decoupling of the molecules from the supporting substrate is therefore desirable [3], if not mandatory.

In this Letter, we show that insulating films having a thickness of only few atomic layers on a metallic substrate provide a sufficient electronic decoupling to allow the inherent electronic properties of individual molecules to be studied. At the same time, the electrons can still tunnel through the ultrathin insulating films, facilitating imaging with the low-temperature STM at a low tunneling current. As an exemplary case, we studied individual pentacene molecules on one, two, and three layers of $\mathrm{NaCl}$ on copper surfaces. STM images acquired at bias voltages corresponding to the negative (NIR) and positive ion resonance (PIR) perfectly resemble the structures of the lowest unoccupied (LUMO) and highest occupied (HOMO) molecular orbital of the free molecule, that is, the bare molecule in vacuum. This opens up the fascinating and unprecedented possibility to obtain direct images of the native molecular orbitals, completely disentangled from the electronic structure of the substrate. This electronic decoupling effect provided by the $\mathrm{NaCl}$ film is further analyzed by elastic scattering quantum chemistry (ESQC) calculations [4].

The experiments were carried out with a home-built low-temperature STM operated at $5 \mathrm{~K}$. $\mathrm{Cu}(111)$ and $\mathrm{Cu}(100)$ single-crystal samples were cleaned by several sputtering and annealing cycles. $\mathrm{NaCl}$ was evaporated thermally, while the sample temperature was kept at 220 to $300 \mathrm{~K}$, so that defect-free, (100)-terminated $\mathrm{NaCl}$ islands of up to three atomic layers were formed [5-7]. $\mathrm{NaCl}$ has a wide band gap of $8.5 \mathrm{eV}$, which has been shown to exist already for a bilayer of $\mathrm{NaCl}$ [8]. Individual molecules were adsorbed at a sample temperature of $T \simeq 5 \mathrm{~K}$, with the sample located in the STM. Bias voltages refer to the sample voltage with respect to the tip. An electrochemically etched tungsten wire was used for the STM tip. All the data discussed were obtained on two layers of $\mathrm{NaCl}$ on $\mathrm{Cu}(111)$, except where stated otherwise.

The influence of any supporting substrate on the STM images of an adsorbed molecule strongly depends on the adsorption site and the resulting symmetry of the combined adsorbate/substrate system. Consequently, a site determination is necessary when discussing STM images of adsorbates. On ultrathin $\mathrm{NaCl}(100)$ layers, atomically resolved images can be obtained $[9,10]$. However, a site determination by a simultaneous imaging of the atomic $\mathrm{NaCl}$ structure and the adsorbed pentacene molecules was not possible, because to image the molecules on the insulating layer requires scanning at a very low tunneling current, so as to prevent the tip from interacting with the molecules during scanning. Instead, a site determination of pentacene on $\mathrm{NaCl}(100)$ was done with the help of coadsorbed gold adatoms as markers, which are known to reside on top of the $\mathrm{Cl}$ ions [7]. As can be seen in Fig. 1, the center of a pentacene molecule is positioned on top of a $\mathrm{Cl}$ ion of the $\mathrm{NaCl}$ layer and the long axis of the molecule is aligned parallel to one of the polar $\langle 011\rangle$ directions of the $\mathrm{NaCl}$ films.

Differential conductance $(d I / d V)$ spectroscopy on an individual pentacene molecule exhibits two distinct features in the $d I / d V$ signal $[11,12]$, centered around -2.4 and $1.7 \mathrm{~V}$, and a broad gap region of low conductance between these peaks (Fig. 2). Bias-dependent imaging shows that directly correlated to the spectroscopic features 


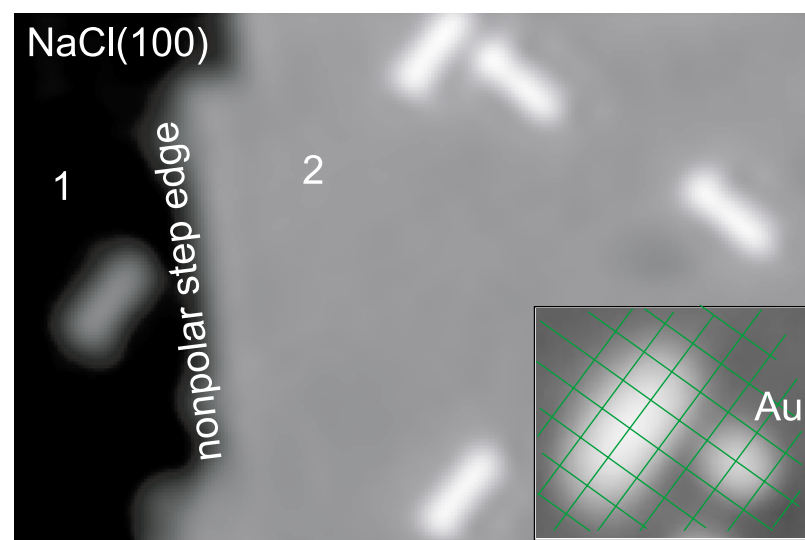

FIG. 1 (color online). Pentacene molecules on one and two layers of $\mathrm{NaCl}$ on $\mathrm{Cu}(111)$. Numbers indicate the layer thickness. The long axis of a pentacene molecule is aligned parallel to one of the polar $\langle 011\rangle$ directions of the $\mathrm{NaCl}(100)$ films, as deduced from the orientation of the nonpolar $\mathrm{NaCl}$ step edge. The center of a pentacene molecule is located on top of a $\mathrm{Cl}$ ion of the $\mathrm{NaCl}$ film, which was determined indirectly with the help of coadsorbed $\mathrm{Au}$ adatoms (inset).

three qualitatively different STM images are obtained: When imaging within the broad gap region, the pentacene molecule appears as a featureless rod of $\simeq 1.2 \AA$ in height, as shown in the upper center panel of Fig. 3. In contrast, for voltages exceeding the peak positions, the molecule appears much broader and higher, and exhibits internal structure (panels to the left and right). The apparent height increases to $\simeq 2.0$ and $\simeq 3.5 \AA$ for voltages below -2.4 and above $1.7 \mathrm{~V}$, respectively. The spatial resolution can be much enhanced by picking up a pentacene molecule with the STM tip (second row of Fig. 3). In this case, the image

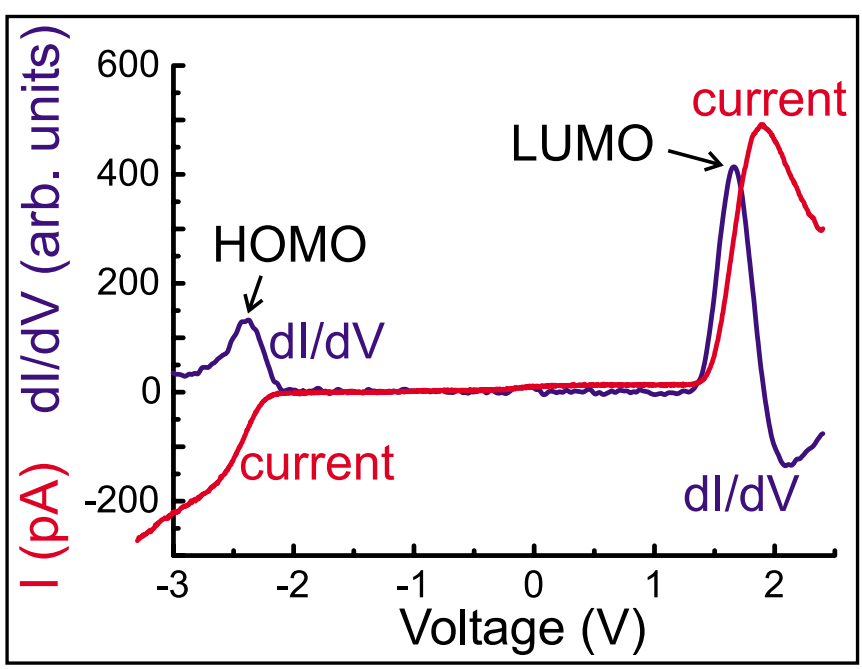

FIG. 2 (color online). $d I / d V$ spectroscopy at the center of a pentacene molecule on $\mathrm{NaCl}$. The $d I / d V$ signal shows two distinct peaks that can be attributed to the NIR and PIR states, and a broad gap of low conductance in between the peaks $[11,12]$. acquired at voltages in the gap region shows five faint protrusions, $\simeq 2.3 \AA$ apart. The corresponding STM images at voltages below -2.4 and above $1.7 \mathrm{~V}$ very closely resemble the native HOMO and LUMO of the free molecule (third row of Fig. 3 and Ref. [13]), which were calculated using the first-principles plane-wave densityfunctional-theory (DFT) program DACAPO [14].

When a molecule is adsorbed onto a metal surface, its orbitals are not only influenced and broadened by a direct coupling to the electronic states of the substrate, but there is also a mutual electronic coupling of different molecular states through the surface $[15,16]$. This effect usually results in such a strong distortion of the molecular states that there is hardly any resemblance to the native orbitals of the free molecule. Adsorbed on $\mathrm{NaCl}$, the pentacene has to interact with the metal surface through the $\mathrm{NaCl}$ layers. The direct correspondence between the experimental STM images and the molecular orbitals of the free molecule clearly demonstrates that the electronic decoupling provided by the insulating film is sufficient to preserve the inherent electronic properties of the free molecule. This opens up the fascinating possibility to directly image the unperturbed molecular orbitals as we have also experimentally observed for tetracene, acridine, and $\alpha-6 \mathrm{~T}$, for example [17].

Apart from that, thin insulating films are also of direct technological interest for devices employing organic molecules. As has been shown in several experiments, thin

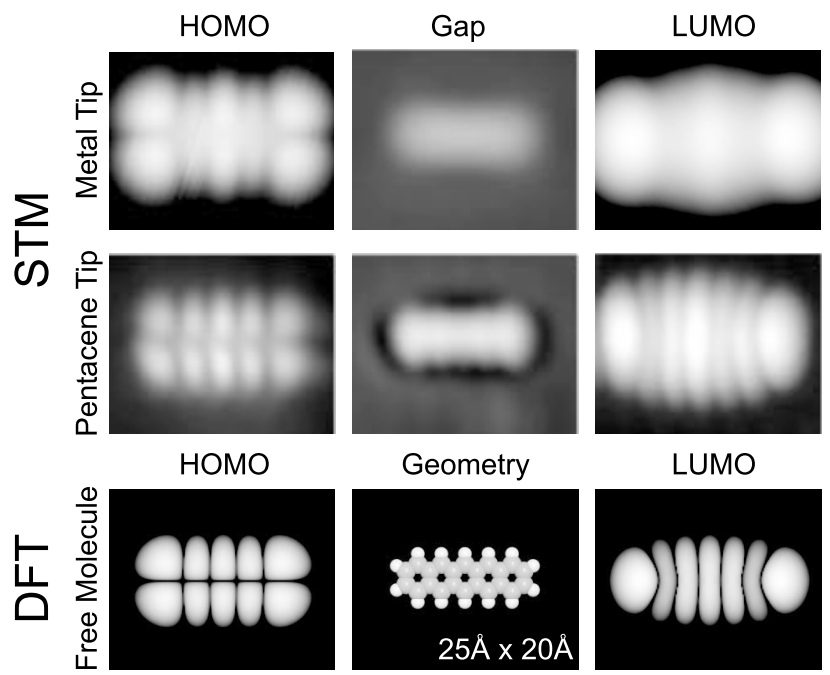

FIG. 3. From top to bottom, the images are STM images acquired with a metal and a pentacene tip, and contours of constant orbital probability distribution $\left(|\Psi|^{2}=5 \times\right.$ $10^{-4} \AA^{-3}$ ) of the free molecule. Whereas the STM images for bias voltages in the HOMO-LUMO band gap are relatively featureless (center), the images at bias voltages exceeding the HOMO (left) or LUMO (right) exhibit very pronounced features, resembling the electron density of the HOMO (left) and LUMO (right) of the free molecule [32]. The geometry of the free pentacene molecule is displayed in the lower center image. 
insulating films can aid in the energetic level alignment and thereby strongly improve device performance $[18,19]$. The detailed understanding of the energetic levels in such structures is therefore of great importance. STM offers the possibility to perform local spectroscopy on an individual molecule in a set of different well-characterized and defect-free structures.

As the work function of $\mathrm{NaCl} / \mathrm{Cu}(111)$ is known to be about $4 \mathrm{eV}$ [20], the peaks in the $d I / d V$ spectrum correspond to a position of the HOMO and the LUMO of 6.4 and $2.3 \mathrm{eV}$ below the vacuum level, respectively. It is important that the lifetime of an electron or a hole on the pentacene molecule is strongly increased by the presence of the insulating layer. An electron or a hole has to localize within the molecule before it can tunnel away into the substrate. Thus, the situation is very similar to the case of electron transport within a molecular solid, where the so-called transport gap is different from the HOMO-LUMO gap of the neutral molecule [1]. The peak positions correspond to the NIR and PIR states of the adsorbed molecule. These states are related to the ionization energy of $6.61 \mathrm{eV}$ $[21,22]$ and the electron affinity of $1.35 \mathrm{eV}$ [23] of the free molecule. These energies are, however, shifted towards the Fermi level because of the electronic polarization of the $\mathrm{NaCl}$ film and the underlying metal [24]. The by far slower ionic polarization of the substrate will take place only after the electron or hole is fully localized on the molecule, and therefore will not decrease the gap size.

According to the Franck-Condon principle, some part of the electron energy is transferred into vibrational energy within the pentacene molecule $[24,25]$. This energy is in the range of about $0.05 \mathrm{eV}$ [21]. The large width of the peaks, which is larger than the vibrational energies of the relevant modes [21], prevents a direct observation of this coupling in the $d I / d V$ spectra [25].

When varying the current in the range of $7.5 \mathrm{pA}$ to $1.1 \mathrm{nA}$, which is accompanied by a change in the tipsample distance of about $2 \AA$, the LUMO peak is shifted by less than $20 \mathrm{mV}$, or about $1 \%$ of the applied bias voltage. This observation suggests that in this current range the influence of the applied electric field on the peak positions (Stark shift) is rather small. By increasing the $\mathrm{NaCl}$ layer thickness, the electronic polarization of the metal substrate can be reduced and directly correlated to the observed width of the gap, which is 3.3, 4.1, and $4.4 \mathrm{eV}$ for one, two, and three $\mathrm{NaCl}$ layers, respectively. When changing the metal substrate orientation from $\mathrm{Cu}(111)$ to $\mathrm{Cu}(100)$, we observe an almost identical gap width for a bilayer of $\mathrm{NaCl}$, but an overall shift of the peak positions of $\simeq$ $0.35 \mathrm{eV}$ towards lower voltages. This compares nicely with the difference in work functions, which for the clean surfaces measures $\Delta \Phi \simeq 0.35 \mathrm{eV}$ [26]. This observation suggests that the molecular levels of the pentacene molecule are coupled to the vacuum level of the supporting $\mathrm{NaCl} / \mathrm{Cu}$ system. This is yet another indication of the electronic decoupling provided by the $\mathrm{NaCl}$ film: In the case of $\pi$-conjugated molecular films directly adsorbed onto a metal surface, a variety of interface phenomena can be observed that cause the breakdown of the vaccum level alignment [27]. In contrast, for weakly interacting adsorbates such as Xe atoms, a vacuum level alignment is observed and can even be used to measure the local work function of many metallic substrates [28].

Whereas the overall resemblance of the STM images with the orbitals of the free molecule is directly evident, a quantification of a possible influence of the $\mathrm{NaCl}$ layer on the imaging and of the natural line width of the molecular resonances require a theoretical study. To determine the influence of the $\mathrm{NaCl}$ film in detail, we calculated the corresponding STM images using ESQC. In our calculations, the full electronic structure of the tip apexpentacene- $\mathrm{NaCl} / \mathrm{Cu}(111)$ tunneling junction is taken into account. In ESQC, the tunneling current is calculated by considering the junction as a multichannel electronic scattering center for electrons coming from the bulk of the STM tip or from the substrate [4]. The electronic levels and molecular orbitals of this tunneling junction are calculated using the semiempirical extended Hückel (EHMO) approximation [29]. The EHMO parametrization for the $\mathrm{NaCl}$ layer was done by calculating its band structure using the BICON-CEDIT program [30] and comparing it with the experimental one [31]. The geometrical structure of the $\mathrm{NaCl}$ bilayer in our calculations is bulklike and rigid, with the lower $\mathrm{NaCl}$ layer $3.0 \AA$ above the topmost $\mathrm{Cu}(111)$ surface layer. The pentacene molecule was kept planar $3.0 \AA$ above the upper atomic layer of the $\mathrm{NaCl}$ film with its lateral position as deduced from the experimental site determination (cf. Fig. 3). In the calculations, which do not include coupling to phonons, we find that the width of the Lorentzian-shaped HOMO and LUMO resonances of the pentacene molecule is on the order of only a few hundreds of micro eV [11], which is equivalent to a lifetime of a hole or an electron in the HOMO or the LUMO on the order of a few picoseconds. As can be seen from Fig. 4, the simulated STM images for a tunneling current of $1 \mathrm{pA}$ at energies in resonance with the HOMO and the LUMO reproduce the experimental images obtained with the metal tip, including the enhancement of the center and outmost protrusions. When increasing the tunneling current to $100 \mathrm{pA}$, the calculated images correspond to the experimental images obtained with the pentacene tip, even though the pentacene molecule at the tip was not included in the calculations.

To find out whether the enhancement of the center and outmost protrusions at low current is caused by the $\mathrm{NaCl}$ film, we removed the $\mathrm{NaCl}$ bilayer in a separate calculation and adjusted the molecule-metal separation in such a way that the electronic molecule-metal coupling was as strong as through a bilayer of $\mathrm{NaCl}$. The resulting simulated images show a very similar intramolecular corrugation and just the same current-dependent enhancement of the center and outmost protrusions as with $\mathrm{NaCl}$. 

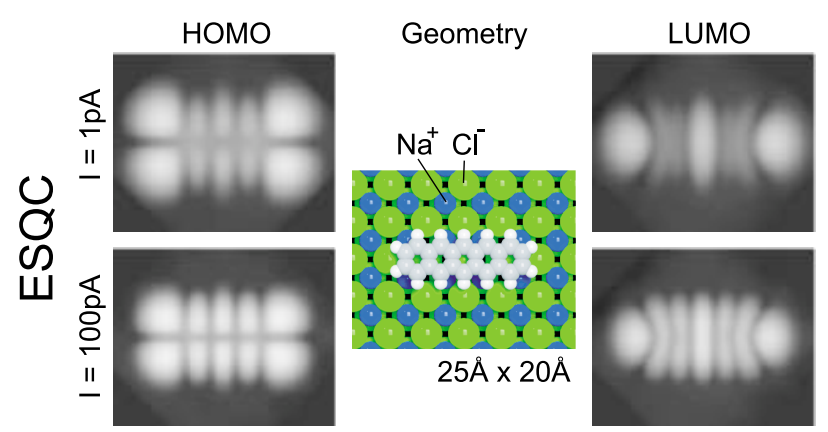

FIG. 4 (color online). Calculated images of the pentacene molecule adsorbed on $\mathrm{NaCl} / \mathrm{Cu}(111)$ using ESQC. The left (right) images are calculated at the energy corresponding to the HOMO (LUMO) of the adsorbed molecule. The images for a current of 1 (upper panel) and $100 \mathrm{pA}$ (lower panel) correspond nicely to the experimental images acquired with the metal and pentacene tip, respectively. In the center the geometry of the adsorbed molecule is shown.

In summary, we have shown that ultrathin insulating $\mathrm{NaCl}$ films disentangle the electronic structure of adsorbed molecules from the influence of the substrate, and thereby open up the unique possibility to directly image the unperturbed molecular orbitals by means of STM.

We acknowledge partial funding by the EU projects "CHIC," "AMMIST," and "NANOSPECTRA." J.R. and G. M. are grateful to Per Hyldgaard for his help on the use of DACAPO and to Heike Riel, Stefan Fölsch, and Mats Persson for valuable comments.

[1] M. Pope and Ch. E. Swenberg, Electronic Processes in Organic Crystals and Polymers (Oxford University Press, Oxford, 1999).

[2] C. Joachim, J. K. Gimzewski, and A. Aviram, Nature (London) 408, 541 (2000).

[3] V. J. Langlais, R. R. Schlittler, H. Tang, A. Gourdon, C. Joachim, and J. K. Gimzewski, Phys. Rev. Lett. 83, 2809 (1999).

[4] P. Sautet and C. Joachim, Chem. Phys. Lett. 185, 23 (1991).

[5] R. Bennewitz, V. Barwich, M. Bammerlin, C. Loppacher, M. Guggisberg, A. Baratoff, E. Meyer, and H.-J. Güntherodt, Surf. Sci. 438, 289 (1999).

[6] J. Repp, G. Meyer, and K.-H. Rieder, Phys. Rev. Lett. 92, 036803 (2004).

[7] J. Repp, G. Meyer, F. E. Olsson, and M. Persson, Science 305, 493 (2004).

[8] S. Fölsch, Ph.D. thesis, Hannover University, Germany, 1991.

[9] W. Hebenstreit, J. Redinger, Z. Horozova, M. Schmid, R. Podloucky, and P. Varga, Surf. Sci. 424, L321 (1999).

[10] J. Repp, S. Fölsch, G. Meyer, and K.-H. Rieder, Phys. Rev. Lett. 86, 252 (2001).

[11] The width of the peaks in the experimental $d I / d V$ spectra of $\simeq 0.3 \mathrm{~V}$ (full width at half maximum) is not limited by electronic interactions with the substrate but by the interaction of the NIR and PIR states with phonons in the insulating layer, as will be discussed elsewhere. Consequently, the shape of the peaks is not Lorentzian but Gaussian-like, and the width is independent of the $\mathrm{NaCl}$ layer thickness as revealed by experiment.

[12] Directly above the LUMO peak there is a region of negative differential conductance. It is important to note that this is not an intrinsic electronic feature of the pentacence $/ \mathrm{NaCl} / \mathrm{Cu}$ system but is due to the measurement. It can simply be understood when considering that the barrier height for the electrons tunneling directly into the LUMO increases with increasing voltage.

[13] R. G. Endres, C. Y. Fong, G. Witte, and Ch. Wöll, Comput. Mater. Sci. 29, 362 (2004).

[14] Computer code DACAPO (CAMP, Technical University of Denmark, Lyngby, Denmark, http://www.fysik.dtu.dk/ campos, 2002) version 2.6.1 using GGA(PW91), a $26 \times$ $17 \times 13 \AA^{3}$ unit cell, $400 \mathrm{eV}$ plane-wave cutoff energy, and $0.05 \mathrm{eV} / \AA$ residual forces.

[15] C. Chavy, C. Joachim, and A. Altibelli, Chem. Phys. Lett. 214, 569 (1993).

[16] J. Lagoute, K. Kanisawa, and S. Fölsch, Phys. Rev. B 70, 245415 (2004).

[17] J. Repp and G. Meyer (unpublished).

[18] S. J. Kang, D. S. Park, S. Y. Kim, C. N. Whang, K. Jeong, and S. Im, Appl. Phys. Lett. 81, 2581 (2002).

[19] L. S. Hung, C. W. Tang, and M. G. Mason, Appl. Phys. Lett. 70, 152 (1997).

[20] R. Bennewitz, M. Bammerlin, M. Guggisberg, C. Loppacher, A. Baratoff, E. Meyer, and H.-J. Güntherodt, Surf. Interface Anal. 27, 462 (1999).

[21] V. Coropceanu, M. Malagoli, D. A. da Silva Filho, N. E. Gruhn, T. G. Bill, and J.L. Brédas, Phys. Rev. Lett. 89, 275503 (2002).

[22] W. Schmidt, J. Chem. Phys. 66, 828 (1977).

[23] L. Crocker, T. Wang, and P. Kebarle, J. Am. Chem. Soc. 115, 7818 (1993).

[24] T. M. Halasinski, D. M. Hudgins, F. Salama, L. J. Allamandola, and T. Bally, J. Phys. Chem. A 104, 7484 (2000).

[25] X. H. Qiu, G. V. Nazin, and W. Ho, Phys. Rev. Lett. 92, 206102 (2004).

[26] P. O. Gartland, Phys. Norv. 6, 201 (1972).

[27] A. Kahn, N. Koch, and W. Gao, J. Polym. Sci., B Polym. Phys. 41, 2529 (2003).

[28] K. Wandelt, Appl. Surf. Sci. 111, 1 (1997).

[29] R. Hoffmann, J. Chem. Phys. 39, 1397 (1963).

[30] M. Brändle, R. Rytz, and G. Calzaferri, computer code BICON-CEDIT, Department of Chemistry and Biochemistry, University of Bern, Switzerland, 2000 (http://iacrs1.unibe.ch).

[31] D. M. Roessler and W.C. Walker, Phys. Rev. 166, 599 (1968).

[32] The images in Fig. 3 have been acquired at $-2300 \mathrm{mV}$, 15 pA (HOMO, metal tip); $429 \mathrm{mV}, 28$ pA (gap, metal tip); $1700 \mathrm{mV}, 15 \mathrm{pA}$ (LUMO, metal tip); $-2523 \mathrm{mV}$, 22 pA (HOMO, pentacene tip); $500 \mathrm{mV}, 22$ pA (gap, pentacene tip); and $1793 \mathrm{mV}, 9.6 \mathrm{pA}$ (LUMO, pentacene tip). 